\title{
Geometrical and Morphological Analysis of Vascular Branches from Fundus Retinal Images
}

\author{
M. Elena Martínez-Pérez ${ }^{1}$, Alun D. Hughes ${ }^{2}$, Alice V. Stanton ${ }^{2}$, \\ Simon A. Thom ${ }^{2}$, Neil Chapman ${ }^{2}$, Anil A. Bharath ${ }^{1}$, and Kim H. Parker ${ }^{1}$ \\ 1 Department of Biological and Medical Systems. \\ Imperial College of Science, Technology and Medicine. \\ Prince Consort Road, London, SW7 2BY, UK \\ 2 Department of Clinical Pharmacology, NHLI, \\ Imperial College of Science, Technology and Medicine, St Mary's Hospital \\ South Wharf Road, London W2 1NY, UK \\ elena.martinez@ic.ac.uk
}

\begin{abstract}
A semi-automatic method to measure and quantify geometrical and topological properties of complete vascular trees in fundus images is described. The method is validated by comparing automatic vs. manual measurements in 17 individual bifurcations. We also compared automatic analyses of complete vascular trees from 4 pairs of red-free and fluorescein images. Preliminary results comparing 10 hypertensive and 10 normotensive subjects show changes in geometrical properties similar to those reported in previous studies. Several topological indices show differences in the arterial, but not venous, trees in hypertensive subjects. This suggests that a combination of geometrical and topological measurements of the whole vascular network may provide more sensitive indicators of morphological changes due to diseases.
\end{abstract}

\section{Introduction}

The basic geometrical properties in branching trees are lengths and diameters of the branches and the angles at which branches meet at bifurcations. Generally both the arteries and veins of the retina are binary trees, whose properties can be considered either locally or globally. The local properties are, for example, the relationships between the branching angles and the ratios of diameters at individual bifurcations. The global properties focus on ordering and relationships between branches. As an example, the orientation of minor branches relative to the anatomical features of the retina could be an interesting topological property.

Retinal blood vessels from fundus images have usually been studied in terms of individual bifurcations, measuring a few of the most clearly visible bifurcations in an image. Differences in geometrical properties in retinal blood vessel have been found as a result of different diseases. Hypertension, for example, causes: reduction in the vessel diameter [8], reduction in the number of vessels present per unit volume of tissue [10], reduction of the branching angle [9] and increase in the length to diameter ratio [3].

In order to study the global properties of branching structures, it has been usual to group bifurcations into schemes based on order or generation. Some 
of these models are ordered from the root of the tree toward the leaves, others from the leaves toward the root. Some of these schemes assume all branches are symmetrical, incorporating diameters and length but not branching angles. Others assume all branches with the same order have the same diameter and length and bifurcate with the same asymmetry. Several studies have been carried out formulating models of the branching geometry, particularly of the human conducting airways, following these different schemes (discussed by [7]). There are no studies, as far as we are aware, related to retinal blood vessels from this global point of view.

In this work a semi-automatic method to measure and quantify geometrical and topological properties of continuous vascular trees in fundus images is described. The combination of geometrical with topological properties in the study of retinal vascular structures could yield more reliable indices to differentiate pathologies in the vascular system.

\section{Vessel Segmentation}

Blood vessels are segmented using a previously described algorithm based on scale-space analysis [5]. Two geometrical features based upon the first and the second derivative of the intensity image along the scale-space, maximum gradient and principal curvature, are obtained by means of Gaussian derivative operators. A multiple pass region growing procedure is used which progressively segments the blood vessels using the feature information together with spatial information about the 8-neighboring pixels. The algorithm works with red-free as well as fluorescein retinal images. Figure 1(a) shows the scanned negative of a red-free retinal photograph and (b) the segmented binary image where the optic disc region is marked in grey, vessels are tracked from this area outwards.

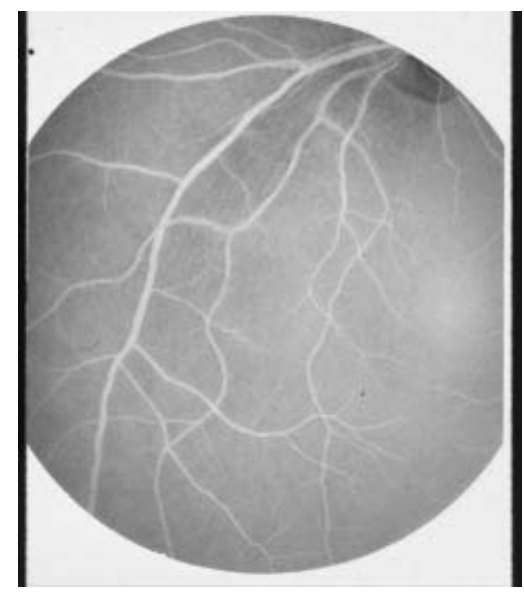

(a)

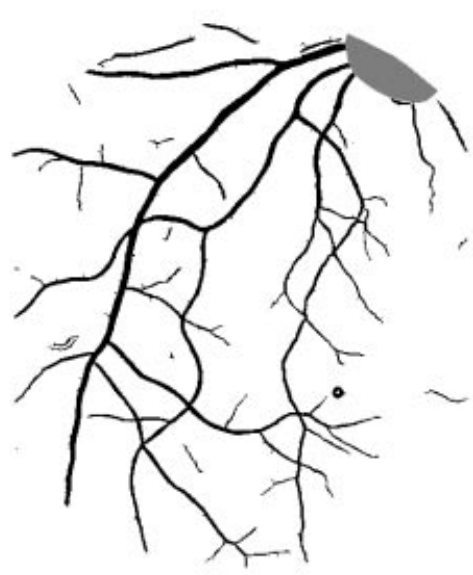

(b)

Fig. 1. (a) Red-free fundus photograph negative, (b) the segmented binary image with the optic disc marked in grey, vessels are tracked from this area outwards. 


\section{Tree Labelling}

Labelling each tree of vessels involves three steps: thinning the segmented binary image to produce its skeleton, detecting significant points and tracking the skeleton of the tree.

Thinning. The skeleton of the vascular tree is obtained from the segmented binary image by a thinning process where pixels are eliminated from the boundaries towards the centre without destroying connectivity in an 8-connected scheme. This is an approximation to the medial axis of the tree. A pruning process is applied to eliminate short, false spurs, due to small undulations in the vessel boundary.

Detecting significant points. Three types of significant points in the skeleton must be detected: terminal points, bifurcation points and vessel crossing points.

In a first pass, skeleton pixels with only one neighbor in a $3 \times 3$ neighborhood are labelled as terminal points and pixels with 3 neighbors are labelled as candidate bifurcation points. Because vessel crossing points appear in the skeleton as two close bifurcation points, a second pass is made using a fixed size window centered at the candidate bifurcations. The number of intersections of the skeleton with the window frame determine whether the point is a bifurcation or a crossing. Figure 2 summarises the three possible cases.

\begin{tabular}{|c|c|c|c|}
\hline Skeleton & $\begin{array}{l}\text { Intersections } \\
\text { with frame }\end{array}$ & $\begin{array}{l}\text { Point } \\
\text { type }\end{array}$ & Output \\
\hline & 2 & Spur & \\
\hline & 3 & Bifurcation & \\
\hline & 4 & Crossing & \\
\hline & & & \\
\hline
\end{tabular}

Fig. 2. Types of significant points.

Once all of the significant points are identified, bifurcation points are labelled as $-r$ where $r$ is the radius of the maximum circle centered on that point that fits inside the boundary of the bifurcation. The sign is used to distinguish between the radius and chain code numbers that are described in the following section. For the crossing points the skeleton is modified to define a single crossing point.

Until this stage the process is fully automatic, but it fails when two true bifurcation points are very close and are merged into a crossing point or when two vessels cross at a very acute angle so that the two candidate bifurcation points fall outside the window frame and are thus defined as two bifurcation points. These cases must be corrected by hand. The complete image is labelled and normally contains several independent vascular trees.

Tracking. Each tree is tracked individually. The coordinates of the starting point of the skeleton of the root are saved and a chain code is generated specifying the direction of the next skeleton point as shown. When the first bifurcation point is reached the chain of the current \begin{tabular}{|l|l|l|}
\hline 4 & 3 & 2 \\
\hline 5 & & 1 \\
\hline 6 & 7 & 8 \\
\hline
\end{tabular} branch is ended and the coordinates of the starting points of the two daughters 
are saved. The process is iteratively repeated for every branch of the tree until a terminal point is reached and all daughters have been numbered. Using a binary scheme, the root is labelled 1 and thereafter the daughters of parent $k_{0}$ are labelled $k_{1}=2 k_{0}$ and $k_{2}=2 k_{0}+1$. With this numbering scheme we are able to track the tree in either direction.

\section{Measurement of Tree Properties}

Using the data generated in the labelling stage and the binary image, three types of geometrical features are automatically measured: lengths, areas and angles.

Lengths. Two lengths are measured directly from the skeleton. The first is the true length of the branch from the starting to the end points of the skeleton computed as $L_{t}=N_{o}+\sqrt{2} N_{e}$ where $N_{o}$ and $N_{e}$ are the number of pixels with odd and even direction codes along the skeleton. The second measure of length is the end-to-end distance $L_{s}$.

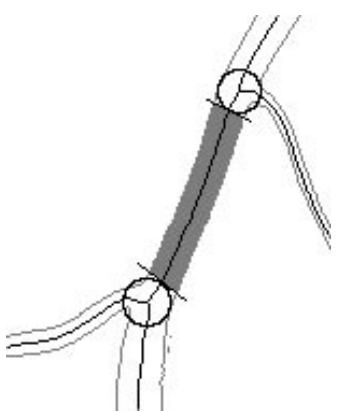

(a)

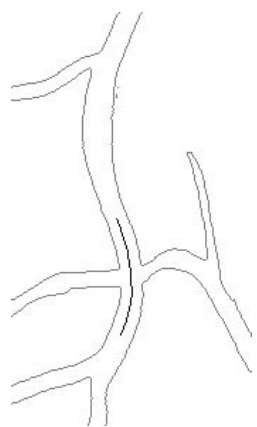

(b)

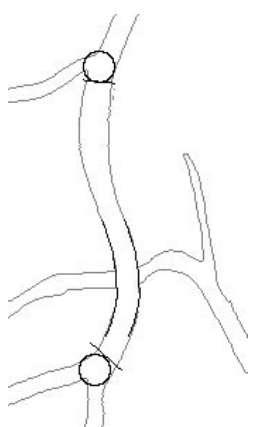

(c)

Fig. 3. (a) The area $A$ is the number of vessel pixels bounded by the two perpendicular lines to the skeleton and $L_{a}$ is its true length. (b) and (c) the borders at crossing points are closed using a displaced section of the skeleton until maximum correlation of pixels with the borders is found.

Areas. Using the value of the radius defined at each bifurcation point, a circle and a line tangent to this circle and perpendicular to the skeleton are drawn on the border image at each end of the branch. This is done to close the region to be measured as illustrated in Figure 3(a). This ensures that ambiguities in the area near bifurcation points are excluded. The number of vessel pixels bounded by these tangents is defined as the area $A$. The average diameter of the selected branch is calculated as $d=A / L_{a}$, where $L_{a}$ is the true length of the segment measured along the skeleton.

When two blood vessels cross, the borders in the crossing region for both vessels are opened. These are closed by taking a section of the skeleton centered at the crossing point and displacing it parallel to the skeleton in both directions 
until maximum correlations of pixels with the border are found (Figure 3(b,c)). This maintains the natural shape of the curvature of the vessel in the crossing region.

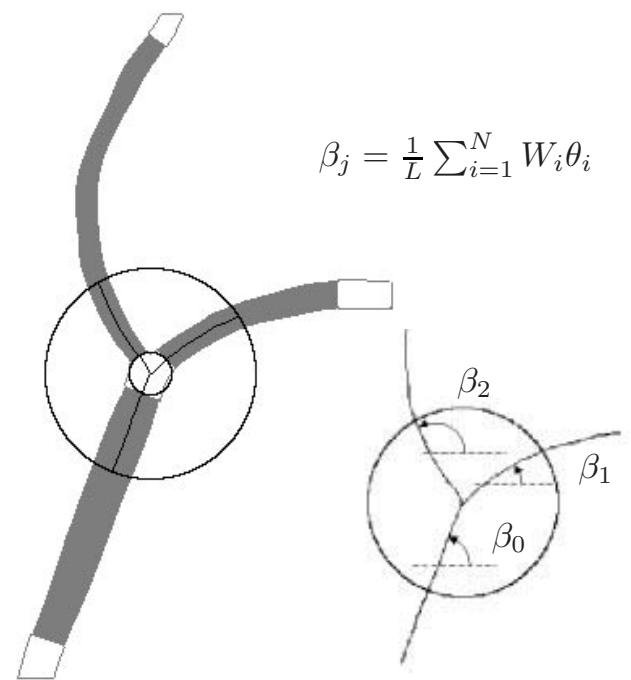

Fig. 4. Three average angles $\beta_{j}$ are defined and measured within a distance of 5 times the radius defined in the bifurcation point.

Angles. A bifurcation angle is associated with a parent vessel. Because a vessel branch can be very tortuous, and because daughters can be parents themselves, three different angles are defined and measured: the total average angle of the branch, a head angle and a tail angle. The latter two are defined within a distance of 5 times the radius of the particular bifurcation point (Figure 4). An average angle is defined as $\beta_{j}=\frac{1}{L} \sum_{i=1}^{N} W_{i} \theta_{i}$, where $\theta_{i}$ is the associated angle of the pixel $i$ ( $45^{\circ}$ steps in the chain code), $W_{i}$ is the associated weight in the chain code $W=1$ for odd and $W=\sqrt{2}$ for even directions, $N$ is the number of pixels in the section of the skeleton and $L$ is the true length of the section.

Derived geometrical properties. Other properties can be derived to describe the vascular tree structure from these measurements.

- Length to Diameter ratio: $\lambda=L_{a} / d$.

- Expansion factor: sum of the cross sectional areas of the two daughter branches divided by the area of the parent $\gamma=\left(d_{1}^{2}+d_{2}^{2}\right) / d_{0}^{2}$.

- Asymmetry factor: the cross sectional area of the minor daughter divided by that of the major $\zeta=d_{2}^{2} / d_{1}^{2}$.

- Angle of daughters: angles of each daughter with respect to the parent $\alpha_{1}=$ $\beta_{1}-\beta_{0}$ and $\alpha_{2}=\beta_{2}-\beta_{0}$.

- Branching angle: $\omega=\alpha_{1}+\alpha_{2}$.

- Angular asymmetry: the angle of the major daughter respect to the parent divided by that of the minor $\eta=\alpha_{1} / \alpha_{2}$.

- Tortuosity: $T=L_{t} / L_{s}$.

- Junction exponent: the exponent $x$ relating the parent and daughter vessel diameters $d_{0}^{x}=d_{1}^{x}+d_{2}^{x}$. Murray [6] suggested that $x=3$ for maximum efficiency in arteries.

\section{Preliminary Results and Conclusions}

Validation of measurements. The true values of geometrical properties in retinal blood vessels remain unknown. Instead indirect methods are used and 
evaluated by comparison with an established technique rather than with the true quantity. With this purpose two validation studies were undertaken. Automatic measurements of individual bifurcations were compared with manual measurements for 17 randomly chosen bifurcations from red-free retinal images. The mean and the standard deviation of the normalised differences of diameter were $-11 \pm 14 \%(n=51, p<0.001)$, i.e. automatic were smaller than manually measured diameters. The normalised differences of branching angles were $13 \pm 12.3 \%(n=17, p<0.006)$, i.e. automatic were larger than manual angles. The $p$ values were calculating using the two sided Wilcoxon signed-rank test [2]. A Runs test for the differences as function of parent vessel diameters showed no systematic tendencies with vessel width. Since the manual measurements involved the average of 5 diameters measured close to the bifurcation and the angles between straight lines fitted by eye, these differences can not be taken as error but as indications of the variability of different measurement techniques.

We also compared automatic measurements of the clinically more common red-free images with automatic measurements of fluorescein images for the same eye in 4 subjects. The normalised differences for the three geometrical features diameters $d$, branching angle $\omega$ and true length of the parent vessel $L_{t}$ (excluding the roots) are shown in Table 1, some of the derived geometrical properties such as length to diameter ratio $\lambda$, tortuosity $T$, expansion factor $\gamma$ and asymmetry factor $\zeta$ are also shown.

\begin{tabular}{|c|c|c|c|}
\hline Feature & Differences (\%) & $n$ & $p$ \\
\hline$d$ & $2.5 \pm 24.5$ & 277 & 0.012 \\
$\omega$ & $-0.1 \pm 12.6$ & 128 & 0.317 \\
$L_{t}$ & $-0.3 \pm 9.1$ & 107 & 0.026 \\
$\lambda$ & $-7.8 \pm 25.8$ & 107 & $<0.001$ \\
$T$ & $0.4 \pm 2.3$ & 107 & 0.073 \\
$\gamma$ & $-11.7 \pm 31.8$ & 128 & $<0.001$ \\
$\zeta$ & $-19.3 \pm 30.5$ & 128 & $<0.001$ \\
\hline
\end{tabular}

Table 1. Mean and standard deviations of the normalised differences of several geometrical features between red-free and fluorescein measurements. Values of $p$ were calculated using the two sided Wilcoxon signed-rank test. Diameter $d$, branching angle $\omega$, true length $L_{t}$, length to diameter ratio $\lambda$, tortuosity $T$, expansion factor $\gamma$ and asymmetry factor $\zeta$. Measures with $n=107$ were done in parent branches excluding roots.

The $p$ values of the Wilcoxon test show that there is no a significant difference between $\omega$ and $T$. The rest of the features were significantly different but the normalised differences are all small and significance is due to the large number of vessels measured. For example, the normalised difference in $d$ corresponds to only $2.5 \%$. The Runs test again showed no significant tendencies, except for the diameters in which there is a tendency to under estimate diameter in vessels with diameters less than 12 pixels. Most of these vessels are terminal branches which were more difficult to detect in the lower contrast red-free images. It should also be noted that the number of bifurcations analysed using the automatic analysis is significantly higher than in any study involving manual measurements. 
Structural analysis. A table of measurements is automatically obtained with the basic geometrical features described above for each tree in an image. At the moment, arterial and venous trees are identified by an expert. Figure 5 shows (a) one arterial tree with its keys marked and (b) one venous tree, both from the image in Figure 1(b).

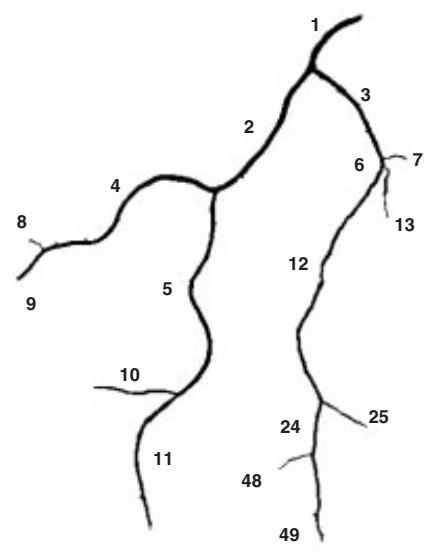

(a)

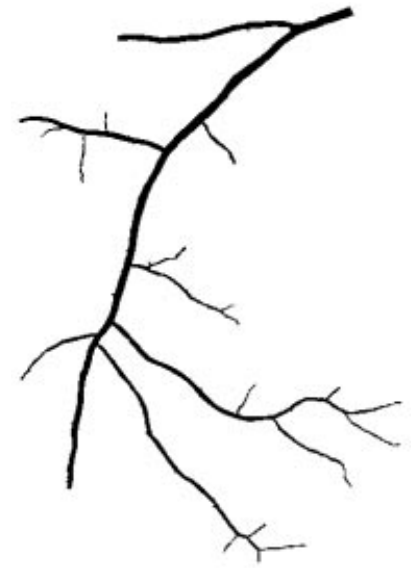

(b)

Fig. 5. Selected trees from Figure 1(b). (a) arterial tree with its vessels numbered and (b) the associated venous tree.

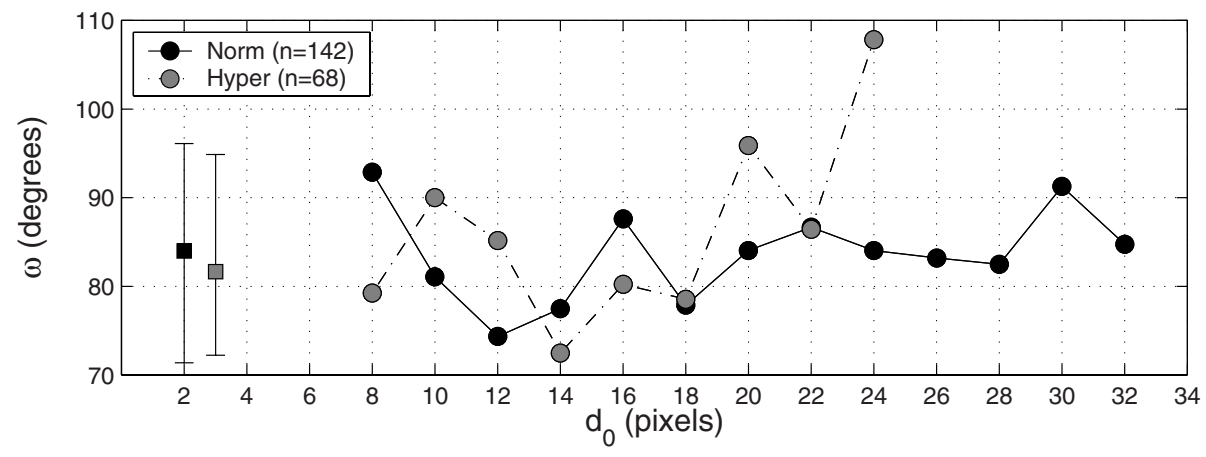

Fig. 6. Median of the branching angle $\omega$ as a function of the parent diameter $d_{0}$ in arterial trees. Normotensive and Hypertensive groups, where $n=$ number of bifurcations. Grand medians and quartiles are to the left (square symbols).

Preliminary results for 10 hypertensive and 10 normotensive subjects using red-free images $(2000 \times 2300$ pixels $)$ have been obtained. A diameter-based classification system, originally used for lung airways [7], is used for our comparisons. Figure 6 shows the branching angle $\omega$ of arterial trees as a function of the parent diameter $d_{0}$. The overall median shows that $\omega$ for hypertensives is more acute than for normotensives as reported in [9], although the difference is 
not significant. In the normotensive group $\omega$ is relatively constant as the parent diameter increases, whereas the hypertensive group shows an increase in $\omega$ for parent vessels $\geq 14$ pixels. A pixel corresponds to approximately $4 \mu \mathrm{m}$ based on the diameter of the optic disc for this scale of images, although due to interindividual variations in refractive indices of the eye it is not possible to establish an absolute scale.

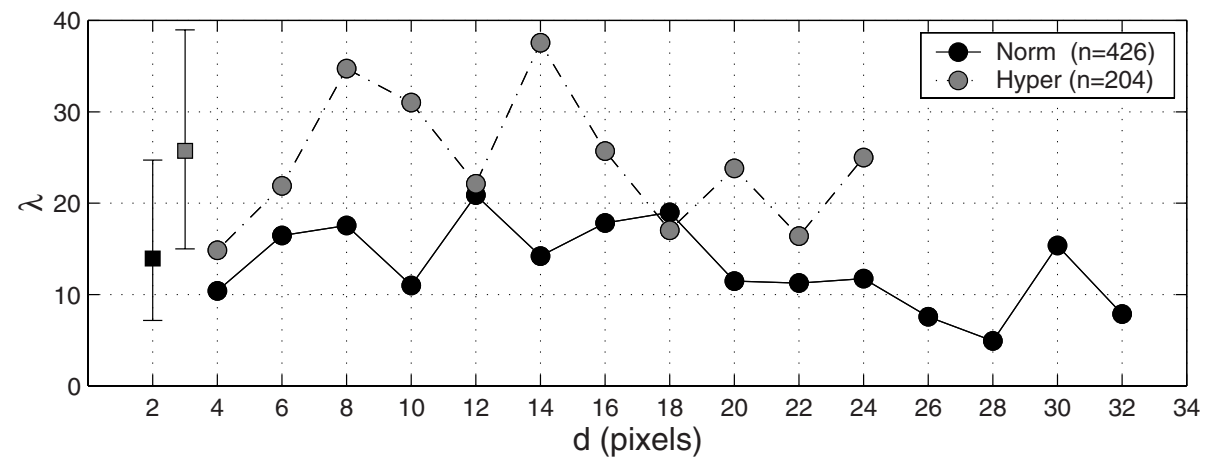

Fig. 7. Median of the length to diameter ratio $\lambda$ as a function of diameter $d$ in arterial trees. Normotensive and Hypertensive groups, where $n=$ number of vessels. Grand medians and quartiles are to the left (square symbols).

Figure 7 shows the median of the length to diameter ratio $\lambda$ of the arterial tree vessels as a function of the diameter $d$. The overall median is significantly larger in hypertensives than normotensives in agreement with [3]. For the normotensive group $\lambda$ is nearly constant over all diameters whereas in the hypertensive group $\lambda$ seems to be larger in the medium sized vessels $7<d<16$ pixels. Note also that the number of vessels in the normotensive group is much higher than in hypertensive, as reported in [10], and that there are no vessels with $d>$ 25 pixels in the hypertensive group, in agreement with the reduction in vessel diameters [8].

Topological analysis. Since we also keep the information about connectivity between branches in the table of measurements that is automatically generated, we are able to make topological analyses of the vascular trees. Vascular trees are branching structures which are treated as binary rooted trees, a special type of graph. A graph is a set of points (or vertices) which are connected by edges. A tree is a graph with no cycles. A rooted tree is a tree in which one vertex is distinguished as the root and a binary tree is one in which at most three edges are contiguous to any vertex [4].

Some important topological features of biological trees are those which measure the symmetry and elongation of a given branching structure. The topological indices we examined in this work are the most commonly used indices within biological applications [1]. (a) Strahler branching ratio $(R)$, (b) maximum tree exterior path length or altitude $(A),(\mathrm{c})$ total exterior path length $\left(P_{e}\right)$, and $(\mathrm{d})$ number of external-internal edges $\left(N_{E I}\right)$, and the total number of external (or terminal) edges $\left(N_{T}\right)$. 
The Strahler branching ratio $(R)$ is calculated by ordering all the edges within a given tree using Strahler ordering. This ordering scheme assigns all external edges an order of one. Where two edges of order $m$ come together, the third edge is assigned to order $m+1$. Where an edge of order $m$ meets an edge of order $n$, the third edge is assigned to order $\max (m, n)$. The Strahler branching ratio $(R)$ is defined as $R=N_{m} / N_{m+1}$, where $N$ is the number of edges and $m$ is the order, Figure 8(a) shows this ordering scheme. The altitude $(A)$ is computed as the largest external path length, where an external path length is defined as the number of edges between the root and the terminal edge. The total path length $\left(P_{e}\right)$ is the sum of all the external path lengths. Figure 8(b) shows an example where $A=6$ and $P_{e}=30$. Finally the number of external-internal edges $\left(N_{E I}\right)$, where an EI edge is an edge which is terminal and its sister is non-terminal, in Figure 8 (c) $N_{E I}=3$ and the total number of terminal edges is $N_{T}=7$. The number of external-external edges $N_{E E}=N_{T}-N_{E I}$.

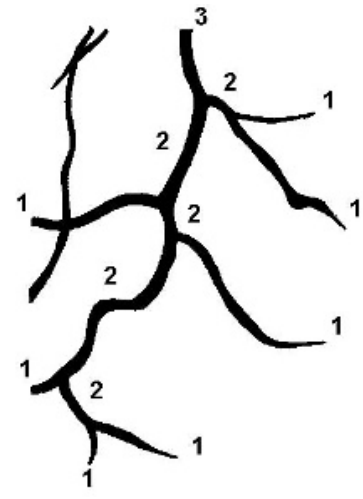

(a)

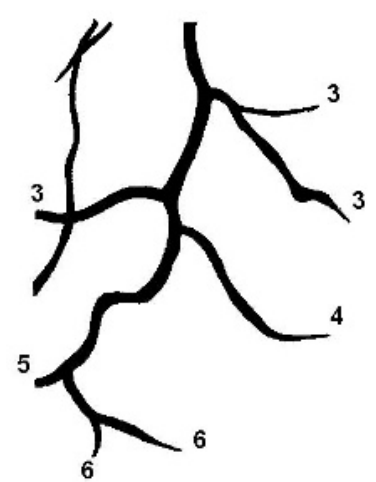

(b)

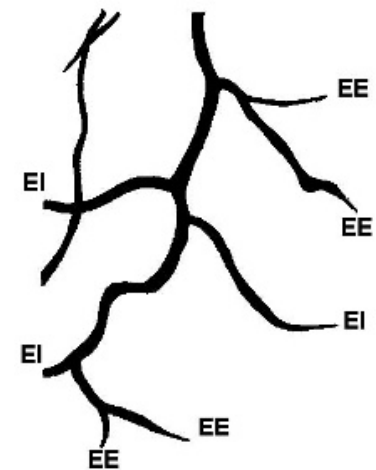

(c)

Fig. 8. Topological indices: (a) Strahler order scheme where the branching ratio is: $R=N_{m} / N_{m+1}$, (b) Altitude $(A=6)$ and total path length $\left(P_{e}=30\right)$, and (c) number of external-internal edges $\left(N_{E I}=3\right)$, where the total number of terminal edges is $N_{T}=7$.

Using the same set of normotensive and hypertensive subjects as before, we calculated each of these topological indices for all trees with $N_{T}>4$, for both artery and vein trees. Table 2 shows the $p$ values for the $t$-test between groups, where we can see that all indices for arteries were significantly different except for the altitude $(A)$, whereas for veins all indices were not significantly different. These results suggest that arteries from the hypertensive group are topologically different from normal. Comparing $R$ for different Strahler orders showed that neither arterial nor venous trees were self-similar.

The purpose of this analysis is not to reach any conclusions about physiology, but to show the kind of analysis that could be usefully applied to provide insights about the changes in the geometry of the whole retinal blood vasculature in diseases such as hypertension. We have shown that the (nearly) automatic method 


\begin{tabular}{|c|c|c|}
\hline Index & Arteries $(n=21)$ & Veins $(n=25)$ \\
\hline$N T$ & $p=0.013$ & $p=0.501$ \\
$A$ & $p=0.247$ & $p=0.385$ \\
$P_{e}$ & $p=0.049$ & $p=0.989$ \\
$E I$ & $p=0.043$ & $p=0.189$ \\
\hline
\end{tabular}

Table 2. Comparison of topological indices between normotensive and hypertensive groups for arteries and veins separately. $p$ values for the $t$-test between groups. Number of terminal edges $N T$, altitude $A$, total path length $P_{e}$ and number of external-internal edges $E I$.

of geometrical quantification proposed in this paper is sensitive enough to show differences in geometrical changes already reported in the literature. It also suggests that analysis of the geometry and topology of the whole vascular network may provide more sensitive indicators of changes due to disease. Future work will include fractal analysis and other combinations of geometrical features and comparisons with other ordering schemes in order to quantify retinal vascular trees more fully.

\section{References}

[1] G.M. Berntson. The characterization of topology: a comparison of four topological indices for rooted binary trees. J. Theor. Biol., 177:271-281, 1995.

[2] J. M. Bland and D. G. Altman. Statistical methods for assessing agreement between two methods of clinical measurement. The Lancet, 1(8):307-310, February 1986.

[3] L.A. King, A.V. Stanton, P.S. Sever, S. Thom, and A.D. Hughes. Arteriolar length-diameter (l:d) ratio: A geometric parameter of the retinal vasculature diagnostic of hypertension. J. Hum. Hypertens., 10:417-418, 1996.

[4] N. MacDonald. Trees and Networks in Biological Models. John Wiley \& Sons, New York, 1983.

[5] M.E. Martínez-Pérez, A.D. Hughes, A.V. Stanton, S.A. Thom, A.A. Bharath, and K.H. Parker. Retinal blood vessel segmentation by means of scale-space analysis and region growing. In C. Taylor and A. Colchester, editors, MICCAI-99, volume 1679 of Lectures Notes in Computer Science, pages 90-97. Springer-Verlag, 1999.

[6] C.D. Murray. The physiological principle of minimum work. i. the vascular system and the cost of blood volume. Proc. Nat. Acad. Sci., 12:207-214, 1926.

[7] C.G. Phillips, S.R. Kaye, and R.C. Schroter. A diameter-based reconstruction of the branching pattern of the human bronchial tree. part i. description and application. Respir. Physiol., 98:193-217, 1994.

[8] A.V. Stanton, P. Mullaney, F. Mee, E.T. O'Brien, and K. O'Malley. A method of quantifying retinal microvascular alterations associated with blood pressure and age. J. Hypertens., 13:41-48, 1995.

[9] A.V. Stanton, B. Wasan, A. Cerutti, S. Ford, R. Marsh, P.P. Sever, S.A. Thom, and A.D. Hughes. Vascular network changes in the retina with age and hypertension. J. Hypertens., 13:1724-1728, 1995.

[10] H.A.J. Struijker, J.L.M. le Noble, M.W.J. Messing, M.S.P. Huijberts, F.A.C. le Noble, and H. van Essen. The microcirculation and hypertension. J. Hypertens., 10(7):S147-S156, 1992. 